\title{
Facile Synthesis of Aluminosilicate
}

\author{
T.T.SUNDARAM ${ }^{1 *}$, N.PRASANNA ${ }^{2}$ and P.JAYAMURUGAN ${ }^{1}$ \\ 'Department of Chemistry, Adhiyamaan College of Engineering, Hosur- 635109 ,Tamil Nadu,India. \\ 'Department of Physics, Adhiyamaan College of Engineering, Hosur - 635109,Tamil Nadu,India. \\ ${ }^{2}$ Department of Chemistry, Government Arts College For Women, Krishnagiri. 635 001,Tamilnadu,India. \\ ${ }^{*}$ Corresponding author Email:sundaramtt@gmail.com \\ http://dx.doi.org/10.13005/ojc/320351
}

(Received: May 24, 2016; Accepted: June 20, 2016)

\begin{abstract}
The researchers in need of an ordered mesoporous material research for the original synthetic protocols. The material was synthesized by one step solvo-thermal crystallization method under acidic conditions using non ionic surfactant as the template. The material was characterized by small angle PXRD,FTIR, and SEM-EDS.
\end{abstract}

Keywords: Aluminiosilicate, small angle PXRD,FTIR ,SEM.

\section{INTRODUCTION}

Though periodic mesoporous silica had been discovered in $1990^{1}$, or even much earlier ${ }^{2}$, this type of materials did not attract much attention until 1992, upon the publication of two groundbreaking papers ${ }^{3,4}$ by a group of Mobil's scientists describing the so-called M41S family of mesoporous silicas. Since then, research on this topic has grown so dramatically that it has developed into a separate field. Remarkable progress has been made in the use of supramolecular templating techniques to synthesize a large variety of periodic inorganic, organic and hybrid mesostructures with tailored framework structures and compositions, pore sizes and architectures, morphologies and surface properties.
Considerable synthesis efforts have been devoted to develop frameworks with pore diameters within the mesoporous range $(2-50 \mathrm{~nm})$ because of their possible use as catalysts, host for inclusion compounds and molecular sieves ${ }^{5}$. Use of the nanosized pores of such host materials to confine the growth of guest materials (semiconductors, metals or compounds) has been widely adopted as an attractive method ${ }^{6}$. The dependence of physical properties of materials on their grain size is a well known phenomenon ${ }^{7,8}$. Due to their small size $(1-100 \mathrm{~nm})$, nanoparticles exhibit novel material properties. However, the engineering of materials on the nanometer scale requires new appropriate synthesis approaches which can provide an effective way of controlling the uniformity of particle sizes and prevent agglomeration of the particles. 
Another important discovery is the use of ordered nanoporous silica and colloidal crystals to create new periodic mesoporous and macroporous materials, including carbons, polymers, metals, and alloys. Combination of different synthesis approaches such as amphiphile, colloidal crystal or microemulsion templating, micro molding and soft lithography led to materials with hierarchically ordered structures. Significant effort was also devoted to the development of potential applications in adsorption, catalysis, separation, environmental cleanup, drug delivery, sensing and optoelectronics.

With the discovery of silicate-based mesoporous molecular sieves using surfactants as templates ${ }^{9}$, a number of research groups have extended this new synthesis strategy to prepare mesoporous AIPOs ${ }^{10-12}$ and SAPOs ${ }^{13-22,27}$. Among the most recent developments in this area is the extension of the amphiphile templating techniques to the synthesis of ordered mesoporous organosilicates and the assembly of zeolite seeds.

\section{MATERIAL AND METHODS}

\section{Material Synthesis}

Ordered mesoporous aluminium silicate was prepared by a combination of surfactant templating, solvo-thermal method, in which nonylphenol was used as a structure-directing agent; anhydrous aluminum chloride and sodium silicate acted as inorganic precursors. In additional mineral acid was added to the synthesis mixture to prevent hydrolysis reaction.

As following, $6.83 \mathrm{~g}$ of anhydrous aluminum chloride was added to a $50 \mathrm{ml}$ of de-ionsed water

Table 1: Elemental composition of material from EDS

\begin{tabular}{lcccc}
\hline Element & $(\mathbf{k e V})$ & Mass\% & Atom\% & K \\
\hline C K & 0.277 & 1.34 & 2.59 & 3.475 \\
O K & 0.525 & 29.72 & 43.19 & 1.3221 \\
Na K & 1.041 & 1 & 1.01 & 0.9924 \\
Al K & 1.486 & 41.31 & 35.6 & 1 \\
Si K & 1.739 & 0.77 & 0.64 & 1.0148 \\
Cl K & 2.621 & 25.86 & 16.96 & 1.3352 \\
Total & & 100 & 100 & \\
\hline
\end{tabular}

and $6.29 \mathrm{~g}$ of sodium silicate under vigorous stirring. When the solution became clear, $4 \mathrm{ml}$ of nonylphenol was added under stirring into the above solution. The mixture was further stirred for 2 to $2.5 \mathrm{~h}$ at $140^{\circ} \mathrm{C}$ to $150^{\circ} \mathrm{C}$. Finally white powdered solid washed with deionized water, and dried at $110^{\circ} \mathrm{C}$.

\section{Characterization}

Powder X-ray diffraction measurements were performed in a Bruker D8 Advance diffractometer using $\mathrm{Cu} \mathrm{K} \alpha$ radiation of wavelength 0.15405 $\mathrm{nm}$. Samples were finely ground before PXRD experiment. Diffraction data were recorded between $2 q=0.5^{\circ}$ to $90^{\circ}$ at an interval of $0.02^{\circ} 2 \theta$.

Fourier-transform infrared (FTIR) spectroscopic investigation was carried out on a Bruker Vector 22 spectrometer using the $\mathrm{KBr}$ pellet.

SEM-EDS electron microscopy images were obtained using a JEOL JSM-6390 SEM operated at $20 \mathrm{kV}$. Samples for analysis were prepared by spreading them on a holey carbon film supported on a copper grid. Energy dispersive X-ray spectroscopy (EDS) was used to investigate the elemental composition of the silicate.

\section{RESULTS AND DISCUSSION}

\section{SEM study of the Aluminosilicate samples}

The surface relief of aluminosilicate is highly heterogeneous due to the coexistence of different aluminosilicate phases together with crystalline and amorphous materials. Crystals of various shapes and size, together with amorphous masses, incorporate into friable grains.

SEM photos taken from as synthesized sample grains are presented in Fig. 1 to provide typical depictions of all the above mentioned crystal types. A nearly homogeneous matrix which is evidenced apart from the clearly visible crystals is attributed to a mixture of meso crystalline phases and amorphous mass ${ }^{23}$. In EDS data (Table 1) shows the material enriched in Al content and Increasing Al content that increase the hydrophilic character of material. Materials with high concentration of $\mathrm{Al}$ increase the selectivity as well as adsorption. 

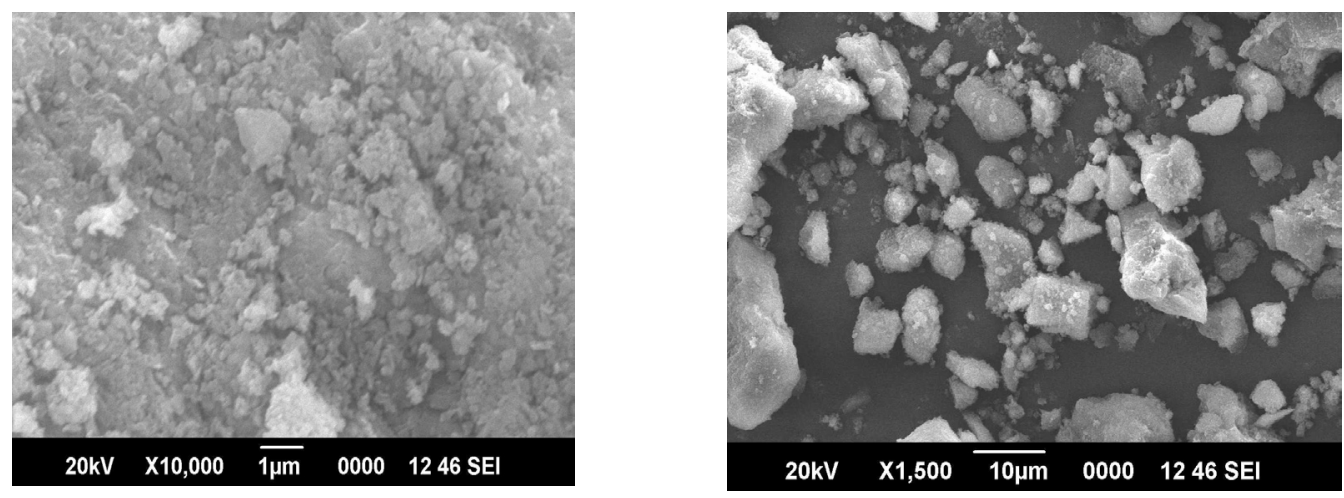

Fig. 1: SEM images of synthesized aluminosilicate

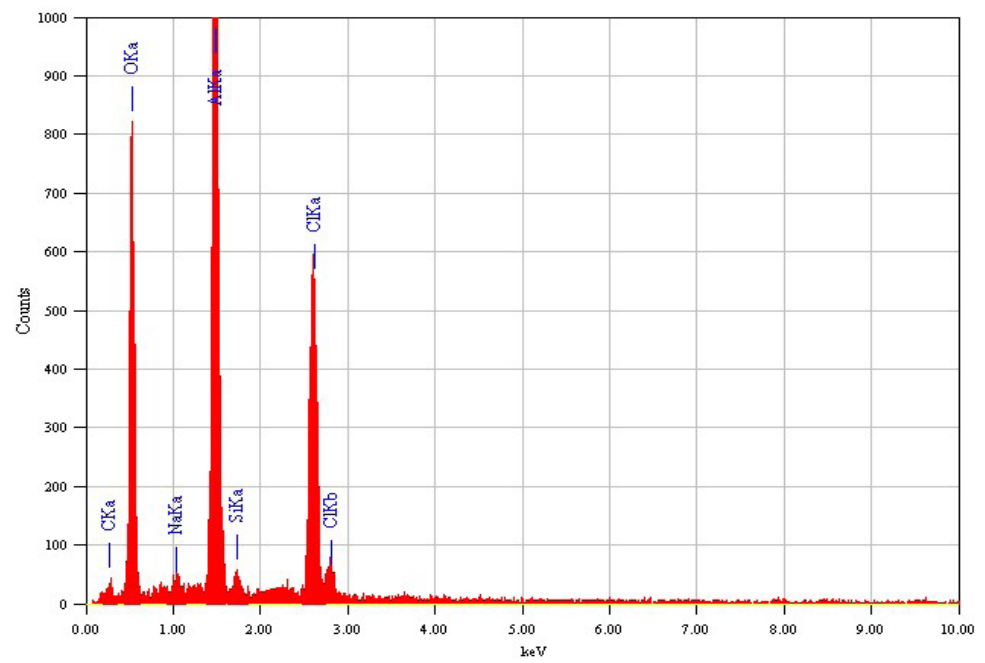

Fig. 2: EDS of synthesized aluminum silicate

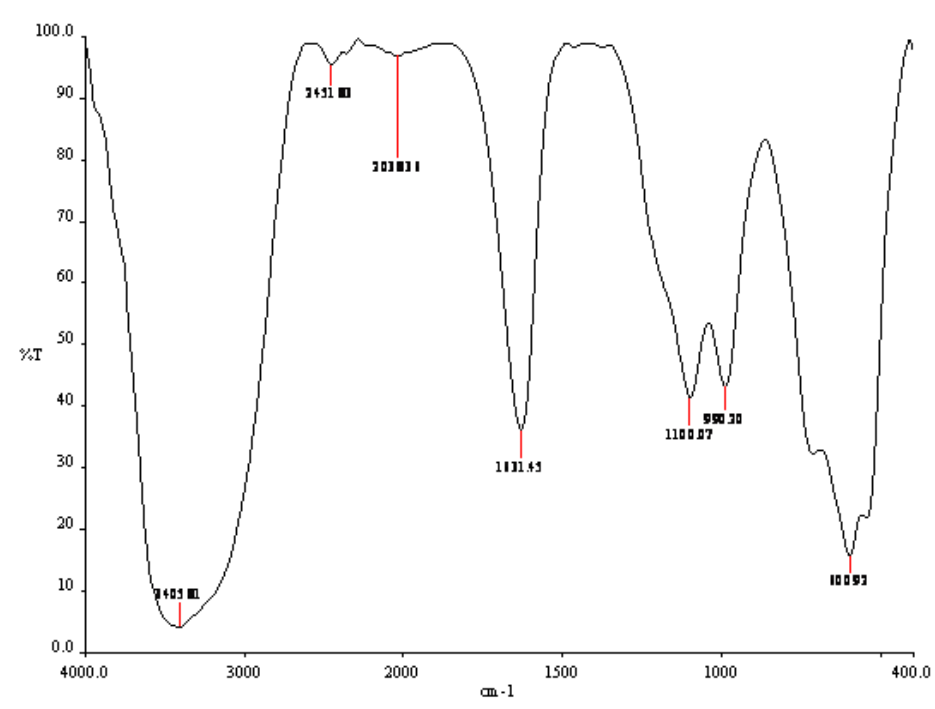

Fig. 3: FTIR Spectrum of synthesized aluminosilicate 


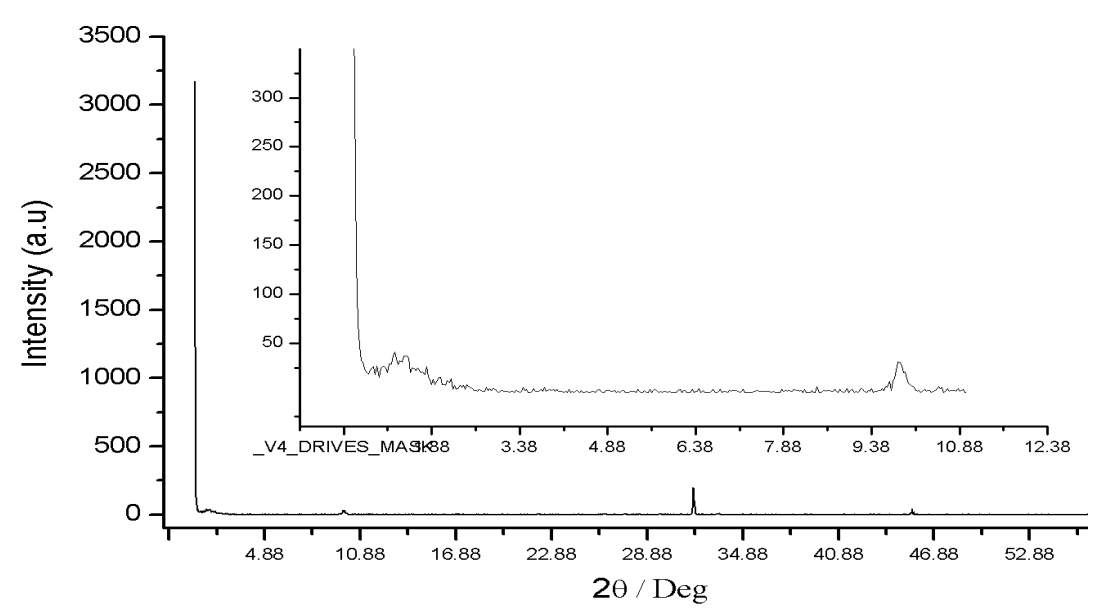

Fig. 4: Small angle PXRD

\section{FTIR study of the Aluminum silicate samples} FTIR spectra can be very useful in obtaining information about the structure of zeolite minerals ${ }^{24,25}$. The vibrations in the region 1600-3700 $\mathrm{cm}^{-1}$ can be assigned to the presence of zeolitic water. The isolated $\mathrm{OH}^{-}$stretching (at $3405 \mathrm{~cm}^{-1}$ in the FTIR pattern of synthesized) is attributed to interaction between the water hydroxyl groups present.

The zeolite phases a strong $\mathrm{T}-\mathrm{O}$ stretching vibration band in the region $1045-1070 \mathrm{~cm}^{-1}$. In synthesized sample this band appears in 1100 $\mathrm{cm}^{-1}$. This band is significant for the estimation of aluminum content in the crystalline framework of aluminosilicate, as it depends on the framework $\mathrm{nSi} / \mathrm{nAl}$ ratio. Band at $1631 \mathrm{~cm}^{-1}$ corresponds to bending vibration of water molecule that is physisorbed in material surface. Band assignment in $950 \mathrm{~cm}^{-1}$ due to condensed $\mathrm{AlO}_{4}$ and band at $600 \mathrm{~cm}^{-1}$ units for $\mathrm{AlO}_{6}$ were interlinked together in material.
Small angle powder X-Ray diffraction provides a measurement pore diameter as long as porosity has a long range repetitive order of material conformed high intense peak at $2 \theta>2^{\circ 26}$.

\section{CONCLUSION}

The results presented show that we were able to prepare high-quality mesoporous aluminosilicate materials with highly thermal stability. The synthesized material was high Al content increase the activity, number active site and adsorption. As synthesized material better for hydrolysis reaction.

It adsorbs moisture from surroundings, so we can be used as a dehydrant and gas sensor applications.

\section{REFERENCES}

1. Yanagisawa T., Shimizu, T., Kuroda, K. and Kato C:'The preparation of AlkyltrimethylammoniumKanemite complexes and their conversion to mesoporous materials', Bull. Chem. Soc. Jpn., 1990, 63,988-992.

2. Di Renzo F., Cambo H. and Dutartre R: '28-Year old synthesis of micelletemplated mesopoorus silica', Microporous Mesoporous
Mater., 1997, 10, 283-286.

3. Kresge C.T., Leonowicz M.E., Roth W.J., Vartuli J.C. and Beck J.S: 'Ordered mesoporous molecular sieves synthesized by a liquid-crystal template mechanism', Nature, 1992,359,710-712.

4. Beck J.S., Vartuli J.C., Roth W.J., Leonowicz M.E., Kresge C.T., Schmitt K.D., Chu, C.T-W., 
Olson D.H., Sheppard E.W., McCullen S.B., Higgins J.B. and Schlenker J.L : 'A new family of mesoporous molecular sieves prepared with liquid crystal templates', J. Am. Chem. Soc., 1992, 114,10834-10843.

5. Huo Q., Margolese D.L., Stucky G.D.: 'Surfactant control of phases in the synthesis of mesoporous silica-based materials', Chem. Mater.,1996, 8,1147- 1160.

6. Zhao D., Feng J., Huo Q., Melosh N., Fredrickson G.H., Chmelka B.F., Stucky G.D.: Science.,1998, 279,548.

7. Gao T., Meng G., Tian Y., Sun S., Liu X., Zhang L., J. Phys.: Condens. Matter., 2002, 14, 12651.

8. Aronson B.B.J., Blanford C., Stein A. :Chem. Mater.,1997, 9, 2842.

9. Li J., Nazer L.F.: Chem. Commun., 2000,1749.

10. Maschmeyer T., Rey F., Sanker G., Thomas J.M.: Nature (London)., 1997, 378,159.

11. Beck J.S., Vartuli J.C., Rith W.J., Leonowicz M.E., Kresge C.T., Schmitt K.D., Chu C.T.W., Olson D.H., Sheppard E.W., McCullen B., Higgins J.B., Schlenker J.L.: J.Am. Chem. Soc.,1992, 114,10834.

12. Oliver S., Kuperman A., Coombs N., Lough A., Ozin G.A.:Nature, 1995, 378, 47.

13. Oliver S., Coombs N., Ozin G.A.: Adv. Mater. 1995, 7, 931.
14. Oliver S., Ozin G.A.: J. Mater. Chem. 1998, 8,1081 .

15. Sayari A., Karra V.R., Reddy J.S., Moudrakovski I.J.: Chem. Commun. 1996, 411.

16. Sayari A., Moudrakovski I.J., Reddy J.S.:Chem. Mater.1996, 8, 2080.

17. Kimura T., Sugahara Y., Kuroda K.: Chem. Lett. 1997, 983.

18. Kimura T., Sugahara Y., Kuroda K.: Micropor. Mesopor. Mater. 1998, 22,115.

19. Kimura T., Sugahara Y., Kuroda K.: Chem. Commun. 1998, 559.

20. Kimura T., Sugahara Y., Kuroda K.: Chem. Mater.,1999, 11.,508.

21. Zhao D., Luan Z., Kevan L.: Chem. Commun. 1997; 1009.

22. Luan Z., Zhao D., He H., Klinowski J., Kevan L., J. Phys. Chem. B, 1998, 102., 1250.

23. Kadlecikova M., Breza J., Jesenak K., Pastorkova K., Luptakova V., Kolmacka M., Vojackova A., Michalka M., Vavra I., Krizanova Z.,: Appl. Surf. Sci. 2008, 254, 5073.

24. Zhao D., Cleare K., Oliver C., Ingram C., Cook D., Szostak R., Kevan L.: Microporous Mesoporous Mater. 1998, 21, 371.

25. Mozgawa W.: J. Mol. Struct. 2001, 596, 129.

26. Solovyov L.A :Chem Sov Rev ,2013,42,3708.

27. Marina G. Xavier, Samuel F. Banda and Christopher C. Chama : Orient J Chem, 2013, 29(4); 1695-1701. 